\title{
Effects of Radial Electric Fields on Linear ITG Instabilities in W7-X and LHD
}

\author{
J. Riemann, R. Kleiber, M. Borchardt \\ Max-Planck-Institut für Plasmaphysik, D-17491 Greifswald, Germany \\ E-mail: joerg.riemann@ipp.mpg.de \\ December 2015
}

\begin{abstract}
.
The impact of radial electric fields on the properties of linear ion-temperaturegradient (ITG) modes in stellarators is studied. Numerical simulations have been carried out with the global particle-in-cell (PIC) code EUTERPE, modelling the behaviour of ITG modes in Wendelstein 7-X and an LHD-like configuration. In general, radial electric fields seem to lead to a reduction of ITG instability growth, which can be related to the action of an induced $E \times B$-drift. Focus is set on the modification of mode properties (frequencies, power spectrum, spatial structure and localization) to understand the observed growth rates as the result of competing stabilizing mechanisms.
\end{abstract}

Keywords: stellarators, gyrokinetics, ITG modes, radial electric fields

\section{Introduction}

As in tokamaks, ion-temperature-gradient (ITG) modes are predicted to be present in stellarators as well. However, their properties can be different and depend on the characteristics of the three-dimensional (3D) magnetic field configuration. With the Large Helical Device (LHD) already working for about 15 years and the Wendelstein 7-X (W7-X) stellarator just commencing its experimental career, there are now two major fusion devices of comparable size available to study the properties of transport in 3D geometry. As W7-X has been optimized for reduced neoclassical transport, anomalous transport is expected to play an important role. This makes the device an interesting object for experimental and theoretical investigation of micro-turbulence and its impact on confinement. Approaching this topic from the modelling point of view, we apply gyrokinetic theory on the level of linear micro-instabilities in order to study the characteristics (growth rates, mode frequencies and spatial structures) of ITG instabilities in 3D geometry.

Numerical simulations are performed with the global gyrokinetic particle-in-cell (PIC) code EUTERPE, which is designed for modelling plasmas in 3D magnetic field 
configurations [1].

One important feature of stellarators is the presence of a neoclassical radial electric field, which is constant on flux surfaces, necessary to fulfill the ambipolarity condition. Depending on the operational regime, this field either points inward (so-called ion-root regime) or outwards (electron-root regime). Both regimes are of interest, but the ionroot regime is the most prevalent in experiments. While the influence of a neoclassical field on the zonal flow residual in stellarators was extensively investigated [2, 3], its effect on ITG modes was rarely studied [4].

Here we summarize the results obtained with simulation of linear ITG instabilities under the influence of radial electric fields applied to both W7-X and LHD-like equilibria. These fields are considered to play a role in suppressing ITG modes in fusion devices, and the investigation of their impact supplements earlier results for ITG instability properties $[1,5,6]$.

\section{Gyrokinetic model equations}

The electrostatic gyrokinetic equation [7] for the ions is solved following a $\delta f$-ansatz for the particle-distribution

$$
f\left(\mathbf{R}, v_{\|}, \mu, t\right)=F_{0}\left(\mathbf{R}, v_{\|}, \mu\right)+\delta f\left(\mathbf{R}, v_{\|}, \mu, t\right),
$$

where the time-dependent part $\delta f$ is assumed to describe small fluctuations around an equilibrium distribution $F_{0}$. The independent variables are the gyro-centre position vector $\mathbf{R}$, the parallel velocity $v_{\|}$and the magnetic moment $\mu$, which is an adiabatic invariant with $\dot{\mu}=0$. The linearized gyrokinetic equation considered here is

$$
\frac{\partial \delta f}{\partial t}+\dot{\mathbf{R}}^{(0)} \cdot \frac{\partial \delta f}{\partial \mathbf{R}}+\dot{v}_{\|}^{(0)} \frac{\partial \delta f}{\partial v_{\|}}=-\dot{\mathbf{R}}^{(1)} \cdot \frac{\partial F_{0}}{\partial \mathbf{R}}-\dot{v}_{\|}^{(1)} \frac{\partial F_{0}}{\partial v_{\|}} .
$$

The unperturbed and perturbed trajectories are given by

$$
\begin{aligned}
& \dot{\mathbf{R}}^{(0)}=v_{\|} \mathbf{b}^{*}+\frac{1}{q B_{\|}^{*}} \mathbf{b} \times\left[\mu \boldsymbol{\nabla} B+q \nabla \Phi_{0}\right] \\
& \dot{v}_{\|}^{(0)}=-\frac{1}{m}\left(\mu \boldsymbol{\nabla} B+q \boldsymbol{\nabla} \Phi_{0}\right) \cdot \mathbf{b}^{*}
\end{aligned}
$$

and

$$
\begin{aligned}
\dot{\mathbf{R}}^{(1)} & =\frac{1}{B_{\|}^{*}} \mathbf{b} \times \nabla\langle\phi\rangle \\
\dot{v}_{\|}(1) & =-\frac{q}{m} \nabla\langle\phi\rangle \cdot \mathbf{b}^{*}
\end{aligned}
$$

with

$$
\mathbf{B}^{*}=\mathbf{B}+\frac{m v_{\|}}{q}(\nabla \times \mathbf{b}) \quad, \quad \mathbf{b}=\frac{\mathbf{B}}{B} \quad, \quad \mathbf{b}^{*}=\frac{\mathbf{B}^{*}}{B_{\|}^{*}} .
$$

Here $m$ and $q$ are the ion mass and charge while $\Phi_{0}$ denotes the external potential,which is assumed constant on each flux surface. 
The quasi-neutrality condition leads, in the long wavelength limit $\left(k_{\perp} \rho_{\mathrm{i}} \ll 1\right)$, to the field equation

$$
-\nabla_{\perp}\left(\frac{m n_{0}}{q B^{2}} \nabla_{\perp} \phi\right)=\left\langle n_{\mathrm{i}}\right\rangle-n_{\mathrm{e}}
$$

determining the perturbed electrostatic potential $\phi$. In our present model, electrons are assumed to respond adiabatically with

$$
n_{e}=n_{0} \frac{e \phi}{k_{\mathrm{B}} T}
$$

The perturbed gyro-averaged density is given by the phase-space integral

$$
\left\langle n_{\mathrm{i}}\right\rangle(\mathbf{x})=\int \delta f \delta(\mathbf{R}+\boldsymbol{\rho}-\mathbf{x}) \mathrm{d}^{6} Z
$$

and

$$
\langle\phi\rangle(\mathbf{R})=\left.\frac{1}{2 \pi} \int_{0}^{2 \pi} \nabla_{\mathbf{x}} \phi\right|_{\mathbf{x}=\mathbf{R}+\boldsymbol{\rho}} \mathrm{d} \xi
$$

defines the gyro-averaged perturbed potential, where $\boldsymbol{\rho}$ and $\xi$ are the gyro-radius vector and the gyro-angle, respectively. [8].

In order to facilitate the treatment of small scale modes, a phase factor transformation is employed. Accordingly, $\phi$ (and analogously $\delta f$ ) is assumed to have the form $\phi=\tilde{\phi} \mathrm{e}^{\mathrm{i}(M \theta+N \varphi)}$, with $\theta$ and $\varphi$ the poloidal and toroidal angular coordinates.

\section{Linear ITG studies with EUTERPE}

\subsection{General outline}

The studies reported in this article are part of a more general approach to the investigation of linear ITG mode properties in stellarator geometry. First results were obtained for the properties of particular ITG modes in W7-X [1]. Subsequent efforts [5, 6] aimed at a systematic documentation of the stability properties of certain configurations over a range of combined temperature and density gradients. The results consistently showed a clear onset of linear ITG mode growth in W7-X as well as in an LHD-like configuration (referred to as LHD from now on) when the (ion) stability parameter

$$
\eta_{\mathrm{i}}=\frac{\mathrm{d} \ln T_{\mathrm{i}}}{\mathrm{d} s} / \frac{\mathrm{d} \ln n_{\mathrm{i}}}{\mathrm{d} s}
$$

exceeded the threshold value $\eta_{\mathrm{i}}=1$. Here, $s=\psi / \psi_{\mathrm{b}}$ denotes the radial coordinate defined by the toroidal flux $\psi$ normalized to its value at the plasma boundary. Successful 'blind test' comparison with the gyrokinetic GENE code strengthened confidence in the simulation results [5]. A basic simplification adopted for these simulations was the use of flat electron temperatures $T_{\mathrm{e}}=$ const in combination with temperature and density profiles determined by

$$
\frac{\mathrm{d} \ln T}{\mathrm{~d} s}=-\sqrt{2}\left(\frac{1}{2}-\left|s-\frac{1}{2}\right|\right) \frac{a}{L_{T}}
$$


and an equivalent relation for the density. The piece-wise linear expression (11) depends on the normalized temperature gradient $a / L_{T}$, measured by the ratio of the average minor plasma radius $a$ ( $a=0.505 \mathrm{~m}$ and $a=0.598 \mathrm{~m}$ for W7-X and LHD, respectively) and the temperature gradient scale length $L_{T}$. The simplified pressure profile resulting from (11) leads to radially localized ITG modes peaking around $s=0.5$, where the instability drive is set to be strongest. This simplification of profiles was chosen in order to benefit from a less complex structure of the excited (global) modes, thus enabling a better analysis of their evolution under the influence of different parameters. Further attention was then paid to the impact of more realistic thermodynamic profiles on ITG mode properties, i.e. growth rates, mode structures, localization, etc. [6].

The main assumptions of the physics model used with the aforementioned studies and the present simulations include: (i) the restriction to the linear phase of ITG growth, (ii) consideration of the electrostatic case only, and (iii) an adiabatic electron response (to enable larger time steps and gain numerical speed-up).

For all our simulations, we employ the concept of 'reference cases' in order to support systematic parameter studies and the comparison of results. According to this concept, we choose arbitrary but particularly well-documented cases from earlier simulations which then provide a starting point for further investigation in different respects.

In the present simulations, we use a standard W7-X case, which is based upon a VMEC equilibrium with $B_{0}=2.41 \mathrm{~T}$ and an assumed pressure profile $p / p_{0}=1-3 s^{2}+2 s^{3}$ corresponding to $\langle\beta\rangle=2 \%$. Temperatures were fixed by $T_{i}=T_{e}=1 \mathrm{keV}$ on the flux surface $s=0.5$, and the normalized temperature gradient in (10) was set to $a / L_{T}=1.41$. For the LHD-like configuration, we consider a model equilibrium with $B_{0}=1.45 \mathrm{~T}$, $p / p_{0}=1-2 s+s^{2}$ and $\langle\beta\rangle=1.5 \%$. Temperatures at $s=0.5$ are again $T_{\mathrm{i}}=T_{\mid} e=1 \mathrm{keV}$, and for this reference case we use $a / L_{T}=4.23$ in (10). Both reference cases assume flat density profiles, corresponding to the most unstable case with $\eta_{i}=\infty$.

Figure 1 shows linear ITG growth rates for W7-X and LHD obtained in a scan over the ion temperature gradient with flat densities and $T_{\mathrm{e}}=$ const. The simulated growth rates for the two devices are similar, although somewhat smaller for LHD with a threshold around $a / L_{T} \approx 1.3$. The data points corresponding to the $a / L_{T}$-values of the two reference cases used in this article are marked.

\subsection{Linear ITG modes with radial electric fields}

As described in [9], radial electric fields in tokamaks can be generated by a net toroidal rotation which modifies the equilibrium particle distribution into a shifted Maxwellian [9]. The amount of rotation, or equivalently the radial electric field, is set by the transport of angular momentum. In contrast, for stellarators radial electric fields are generally smaller than in tokamaks and are set by the ambipolarity condition. In the usual regimes for stellarator experiments, the negative ion-pressure gradient will lead to the inward-oriented radial electric fields of the physically interesting ion-root regime. Under certain assumptions, the details of which are discussed in the paragraph preceding 


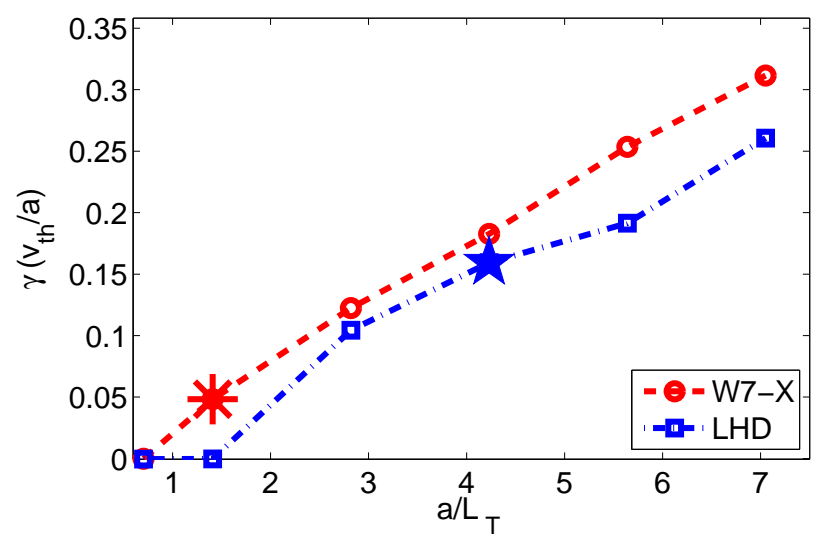

Figure 1. Normalized growth rates of linear ITG instabilities vs. normalized temperature gradient W7-X (dash) and LHD (dash-dot). Values for the present reference cases are marked $(*$ and $\star)$.

eq. (4) of [10], the radial electric field $\mathbf{E}=E_{s} \boldsymbol{\nabla} s$ of the ion-root regime can be estimated from the background ion-pressure gradient $\nabla p_{\mathrm{i}}=p_{\mathrm{i}}^{\prime} \nabla s$ according to [10]

$$
E_{s} \approx \alpha \frac{p_{\mathrm{i}}^{\prime}}{Z_{\mathrm{i}} e n_{\mathrm{i}}} \approx \alpha \frac{\left(n_{e} T_{\mathrm{i}}\right)^{\prime}}{e n_{e}}
$$

where $n_{\mathrm{i}} \approx n_{e}$ and $Z_{\mathrm{i}}=1$ was assumed in the last step. For the purpose of numerical simulation, we also introduced the dimensionless 'strength parameter' $\alpha$ which needs to be $\alpha=+1$ to yield the result derived in [10]. Fields described by eq. (12) will be referred to as 'model A' and will have a two-fold impact on the expected ITG modes. The first one is due to the presence of the field itself while the second one results from its shear $E_{s}^{\prime} / E_{s} \neq 0$ and can draw particular attention to the global aspect of the effects studied. The latter will become clear in comparison with cases that assume un-sheared fields with $E_{s}=\operatorname{const}(\alpha)$, here referred to as 'model B'.

In general, radial electric fields induce $E \times B$-drifts which can reduce the growth of ITG modes, depending on their strength, direction and radial variation. From a common point of view discussed in [4] for both tokamaks and (straight) stellarators, this reduction can occur as result of a shearing mechanism in which the shearing rate of the $E \times B$ drifts overcomes the growth rate of the ITG modes. Reference [4] also describes another stabilizing mechanism for profiles with zero shearing rate, where the position of the maximum mode amplitude is displaced poloidally by the $E \times B$-drift velocity into a region which is less favourable for the instability growth.

The local strength of the radial electric fields is usually measured by the Mach number

$$
M=\frac{\left\langle v_{E \times B}\right\rangle}{v_{\text {th }}} \quad, \quad v_{\text {th }}=\sqrt{\frac{T_{\mathrm{i}}}{m_{\mathrm{i}}}},
$$

given by the induced $E \times B$-drift velocity averaged over the flux surface (here at $s=0.5$ ) and the local ion-sound speed $v_{\text {th }}$. In the present simulations, radial electric field 

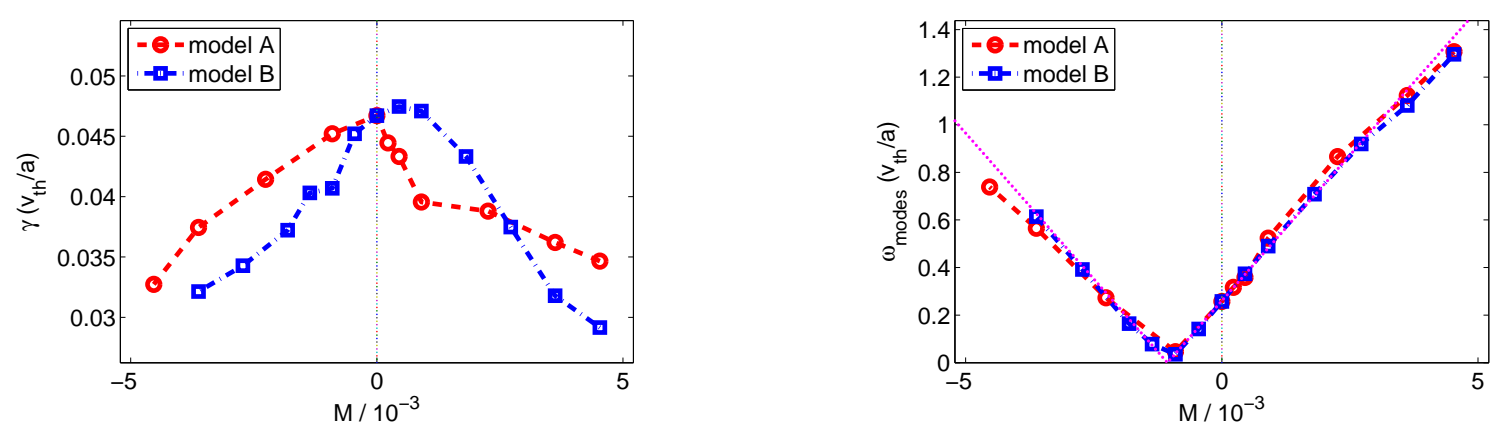

Figure 2. Normalized growth rates (left) and absolute mode frequencies (right) vs. Mach number for W7-X as with model A (dash) and model B (dash-dot) used for $E_{s}$.

strengths have been varied by scanning the strength parameter $\alpha$ through the interval $-1 \leq \alpha \leq+1$. For fields described by eq. (12), $\alpha>0$ corresponds to the ion-root branch with $E_{s}<0$ and Mach numbers $M<0$.

\section{Simulation results}

\subsection{ITG modes in $W^{\text {r}}$-X with radial electric field}

The main results of the simulation runs for the $\mathrm{W} 7-\mathrm{X}$ reference case are summarized in figure 2, displaying the growth rates and frequencies of linear ITG modes in W-7X as functions of the Mach number. The data shows the expected reduction of linear ITG growth by up to $30 \%$ of the value with zero field for both electric field models in the ionroot as well as the electron-root regime. Restricting our analysis to the more relevant ion-root case, we find that the reduction of instability growth is weaker for sheared fields of model A (12) than for constant $E_{s}$ with model B. This may be attributed to the radial variation of $E_{s}$ and thus illustrate the global nature of the observed stabilizing mechanism.

The differences between the two field strength models applied are not reflected in the absolute values of the mode frequencies, which almost perfectly follow a linear dependence and reach $\omega=0$ at $M \approx-10^{-3}$. This suggests that the phase of the observed mode appears Doppler-shifted with respect to the initial propagation along the ion-diamagnetic drift direction. This shift can be interpreted as the result of the induced $E \times B$-drift velocity acting on the ITG mode. Referring to one of the stabilizing mechanisms discussed in [4], this drift velocity could lead to a reduction of growth by pushing the ITG modes poloidally away from their preferred position. To support this picture, we measured the radial and poloidal position of the ITG modes as a function of the Mach number. This position is determined by the 'centre-of-mass' coordinates of the observed mode patterns as they appear in the $s-\theta$-plane at toroidal angle $\varphi=0$. Transforming the poloidal angle $\theta \in[-\pi, \pi]$ according to

$$
\chi=\frac{\theta}{2 \pi}+\frac{1}{2},
$$




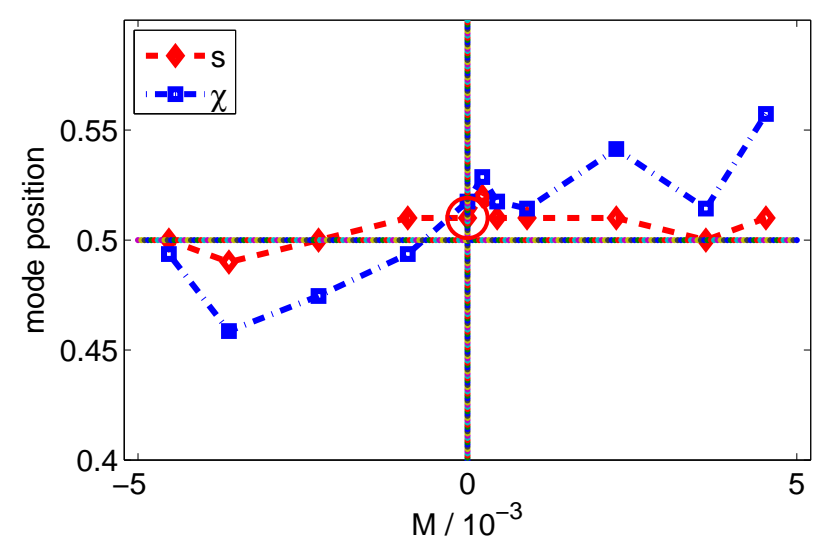

Figure 3. Normalized radial $(s)$ and poloidal $(\chi)$ ITG mode positions vs. $E \times B$-Mach number in W7-X as with model $A$. The circle indicates the radial position with the maximum growth rate for this case.

into the interval $[0,1]$ where $\chi=0.5$ corresponds to a position in the outer equatorial plane of the torus, both radial and poloidal coordinates can be plotted together in one figure.

Figure 3 indeed reveals a (rather) small poloidal downward shift of the ITG modes from $M=0$ to $M<0$, while the modes seem to stay nearly at the same radial position $s \approx 0.5$, where the pressure gradient is set to be strongest. The circle marks the radial position of the mode where the maximum growth rate is measured.

The positions given in figure 3 were obtained from a series of plots like figure 4 , which shows the spatial structure of the ITG modes in the $s$ - $\theta$-plane at $\varphi=0$ for $M=0$ and $M=-4.53 \cdot 10^{-3}(\alpha=+1)$. In addition, figure 5 shows the ITG mode patterns as they appear on a flux surface (here $s=0.5$ ) of one field period in real space. This representation clearly reveals the elongated mode pattern, mainly following the magnetic field lines and avoiding the region of the helical edge [1].

It is worthwhile to note that both figures 4 and 5 show absolute values of the electrostatic perturbation potential $\phi$ normalized to its maximum value, and that the plots for $M=-4.53 \cdot 10^{-3}$ correspond to a mode with clearly reduced growth rate, as seen in figure 2 .

In order to gain deeper insight into the mechanisms that lead to the observed reduction of instability growth, we study the power spectra

$$
\Phi_{m, n}=\int\left|\phi_{m, n}(s)\right|^{2} \mathrm{~d} s
$$

obtained from the local Fourier components of the observed electrostatic perturbation potentials. The left-hand plot of figure 6 shows a power spectrum (at $M=0$ ), which can be regarded characteristic for W7-X as it is very elongated along a line of resonance determined by the relation $n+\iota m=0$, with $\iota$ the rotational transform and $m$ and $n$ 

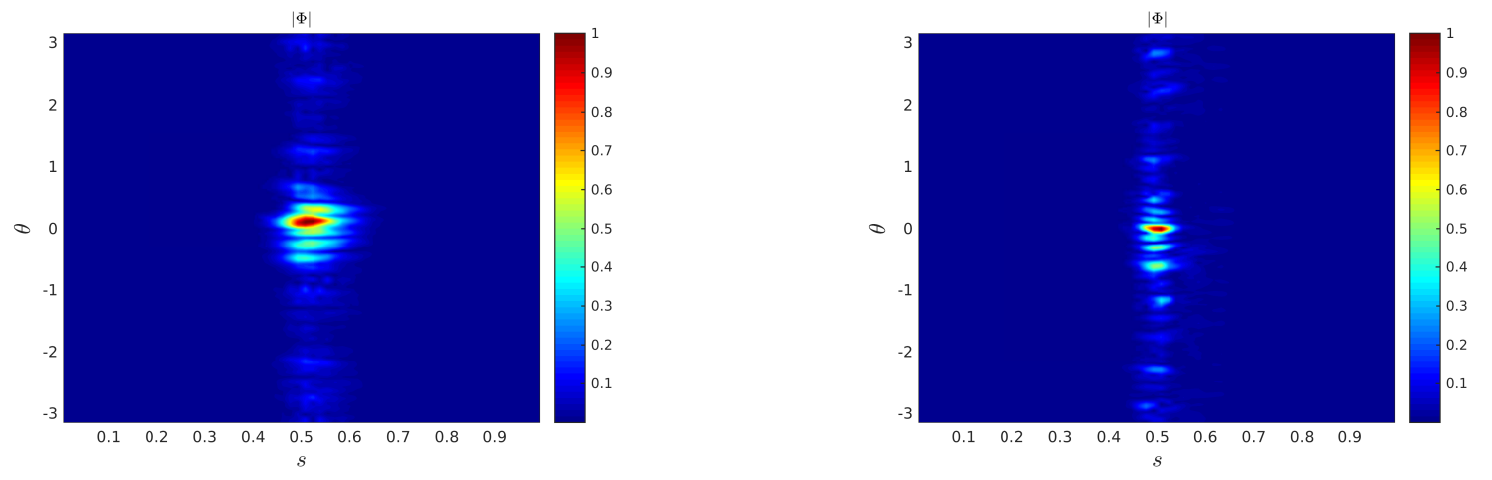

Figure 4. Linear ITG mode structure of the electrostatic potential $\left|\phi / \phi_{\max }\right|$ in the $s$ - $\theta$-plane of W7-X at toroidal angle $\varphi=0$ as with model A for $M=0$ (left) and $M=-4.53 \cdot 10^{-3}$ (right).
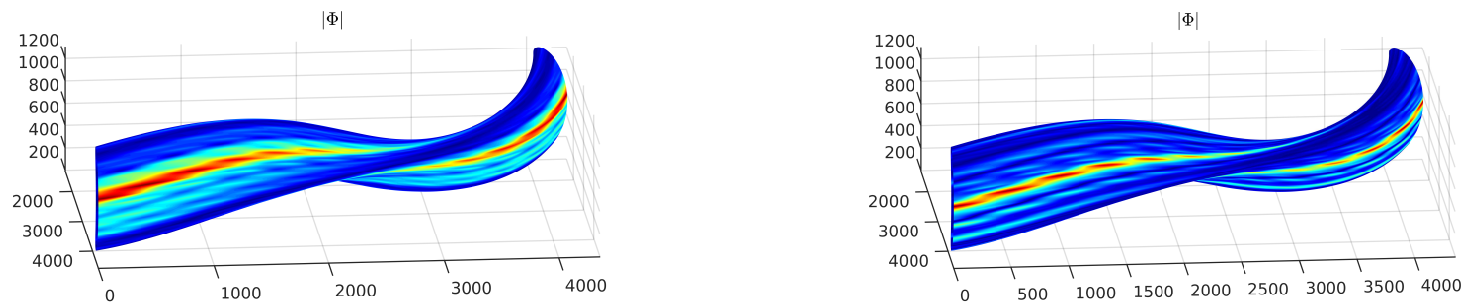

Figure 5. Linear ITG mode structure of the electrostatic potential $\left|\phi / \phi_{\max }\right|$ on the $s=0.5$ flux surface of W7-X as with model A for $M=0$ (left) and $M=-4.53 \cdot 10^{-3}$ (right).

the poloidal and toroidal mode numbers, respectively. In contrast to axially-symmetric systems, the metric properties of 3D-configurations also allow for a coupling between different toroidal modes, which can lead to a Fourier spectrum that occupies a wide region in both $m$ and $n$. The observed elongation and finite width across the line of resonance indicate a relatively strong coupling of poloidal as well as toroidal modes, which may be a consequence of the rather flat $\iota$-profile in combination with the degree of three-dimensionality of $\mathrm{W} 7-\mathrm{X}$. The relatively high mode numbers are due to the relatively low temperature $(1 \mathrm{keV}$ at $s=0.5)$ and correspond to $k_{\perp} \rho \approx 0.75$.

The right-hand plot of figure 6 depicts an ITG mode spectrum which is clearly modified in the presence of an $E \times B$-velocity with $M=-4.53 \cdot 10^{-3}$. The spectrum is still very elongated but now exhibits several spikes which correspond to formerly subdominant Fourier components of comparable magnitude. Moreover, the spectrum has been shifted towards lower mode numbers. Comparing the two cases for $M=0$ and $M=-4.53 \cdot 10^{-3}$, it seems that the original mode was not only spatially displaced (and its growth reduced) but actually modified into a different mode with its own characteristics. Referring to 

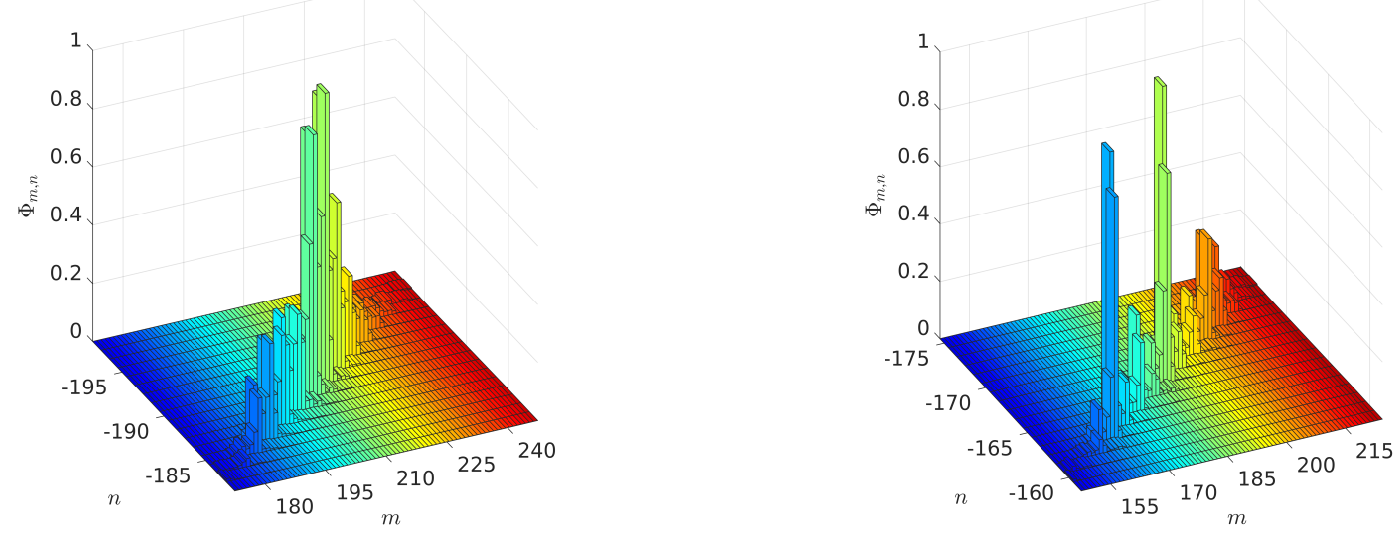

Figure 6. Power spectrum of linear ITG modes in W7-X normalized to their maximum as with model A for $M=0$ (left) and $M=-4.53 \cdot 10^{-3}$ (right).

[4] again, this could be understood as the result of a shearing mechanism that virtually destroys the original ITG mode and allows slower-growing modes to become dominant.

\subsection{ITG modes in LHD with radial electric field}

Applying the same analysis as to $\mathrm{W} 7-\mathrm{X}$, figure 7 shows the growth rates and mode frequencies obtained for our LHD reference case. Again, we find a reduction of growth rates and a Doppler-shift of mode frequencies. For the sheared field model A of eq. (12), we find the growth rates reduced by up to $55 \%$ and $45 \%$ in the ion-root and electron-root cases, respectively. The temperature gradient assumed for the LHD reference case is stronger $\left(a / L_{T}=4.23\right.$ versus $a / L_{T}=1.41$ for $\left.\mathrm{W} 7-\mathrm{X}\right)$, and the observed Mach numbers are correspondingly larger than for the W7-X case. Since electric fields with $E_{s}=$ const are regarded less realistic, simulations have not been performed over the full Mach number range for model B. Aside from the similarities with figure 2 , there are two details in figure 7 that are different and deserve particular attention: (i) The growth rates in the ion-root branch show a slight increase for small field strengths before they drop monotonically. (ii) The absolute mode frequencies are well-described by a linear Doppler-shift within $\pm 8 \cdot 10^{-3}$ around $M \approx-4 \cdot 10^{-3}$ but clearly deviate from this model at higher Mach numbers. These differences can possibly be explained with the help of the diagnostics already employed for the W7-X case.

Figure 8 again shows the radial and poloidal ITG mode positions as a function of the Mach numbers corresponding to $-1 \leq \alpha \leq+1$ in eq. (12). We observe a clear poloidal shift of the ITG mode patterns between $0.3 \leq \chi \leq 0.7$. It seems likely that the magnitude of this shift is a consequence of the stronger fields applied but may also be supported by the geometric properties of the configuration. In particular, LHD does not have a sharply curved helical edge like the one that appears to act as a strong barrier against the poloidal displacement of ITG modes in W7-X [1]. 

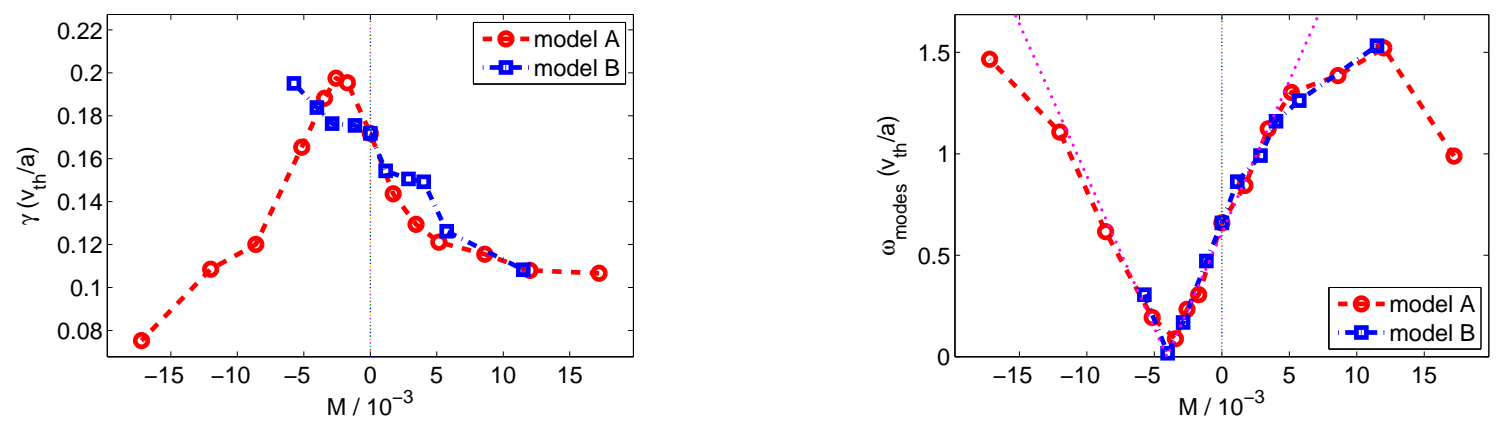

Figure 7. Normalized growth rates (left) and absolute mode frequencies (right) vs. Mach number for LHD as with model A (dash) and model B (dash-dot) used for $E_{s}$.

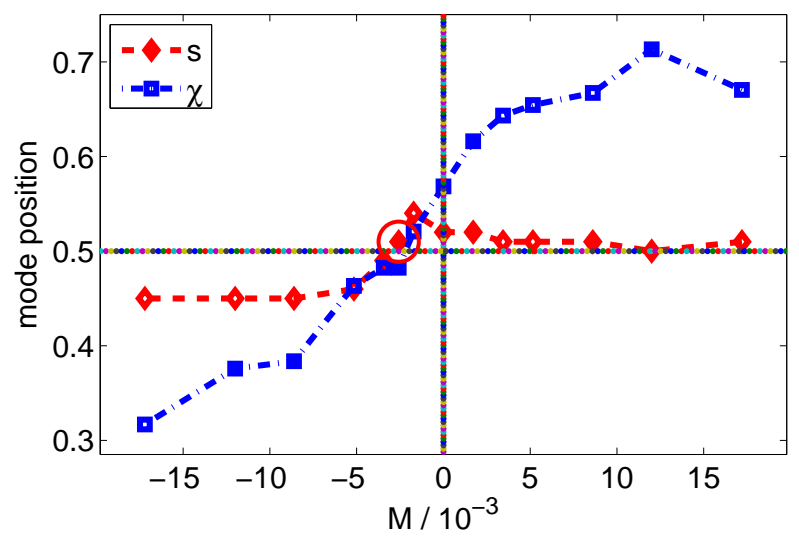

Figure 8. Normalized radial (dash) and poloidal (dash-dot) ITG mode positions vs. $E \times B$-Mach number in LHD as with model $A$. The circle indicates the radial position with the maximum growth rate for this case.

The radial positions given in figure 8 are found to stay nearly at the same flux surface in the electron-root case but show clear excursions in the ion-root regime. The figure also marks the point $M_{\circ} \approx 2.6 \cdot 10^{-3}$ which corresponds to the maximum growth rate measured for this case. At this value, the radial position of the mode is almost exactly at $s=0.5$ and the poloidal position is in the outer midplane. This means that the radially localized pressure profile from eq. (11) with its relatively strong gradient gets combined with the (unfavourable) curvature of the outer midplane to particularly support the growth of the ITG mode. For Mach numbers slightly smaller or larger than $M_{\circ}$, the mode is radially displaced away from $s=0.5$ into regions with smaller pressure gradient, combined with a poloidal shift. For large Mach numbers $M<0$, the modes stay clearly away from $s=0.5$ and are pushed into the lower part of the outboard torus, resulting in strongly reduced growth rates. For large $M>0$, the reduction of grow rates is somewhat weaker since the mode is poloidally displaced, although it remains under the 

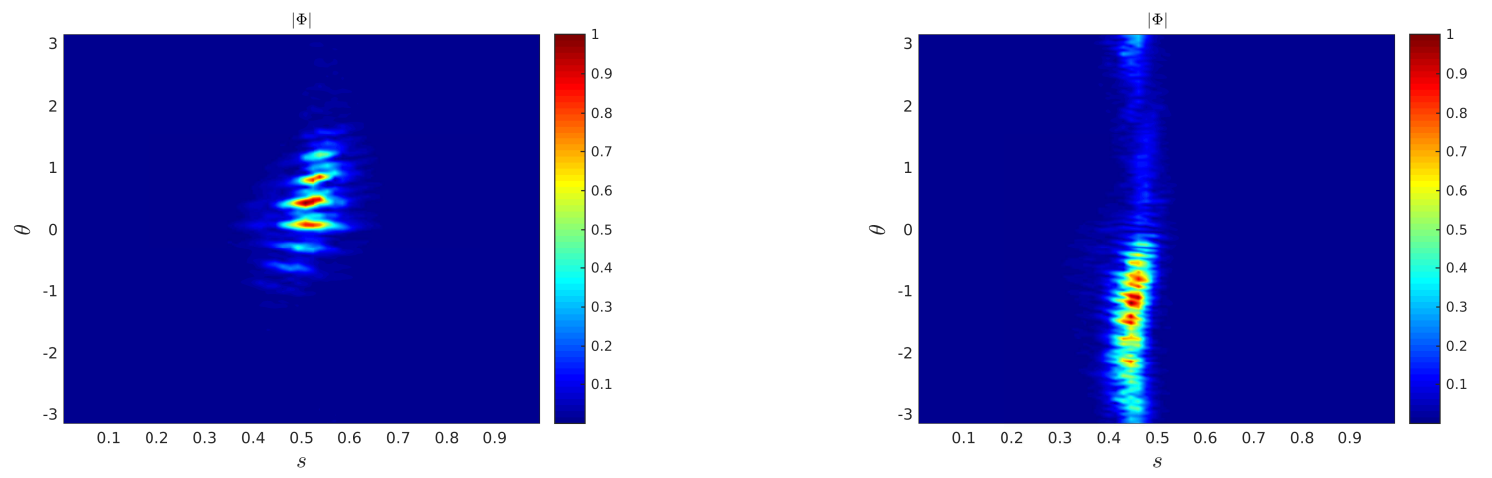

Figure 9. Linear ITG mode structure of the electrostatic potential $\left|\phi / \phi_{\max }\right|$ in the $s$ - $\theta$-plane of LHD at toroidal angle $\varphi=0$ as with model A for $M=0$ (left) and $M=-1.72 \cdot 10^{-2}$ (right).
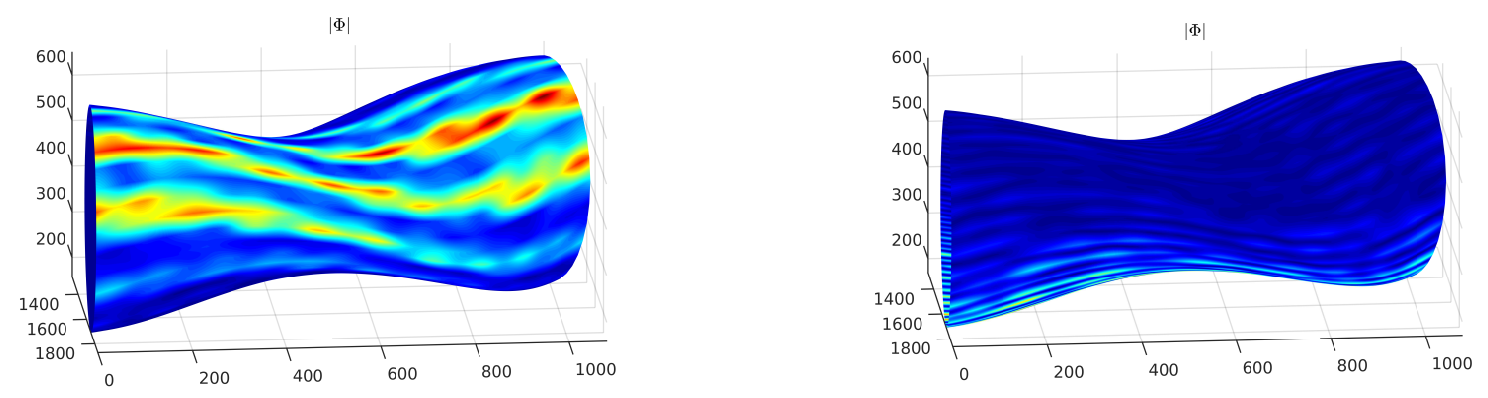

Figure 10. Linear ITG mode structure of the electrostatic potential $\left|\phi / \phi_{\max }\right|$ on the $s=0.5$ (left)ands $=0.45$ (right) surface of LHD as with model A for $M=0$ (left) and $M=-1.72 \cdot 10^{-2}$ (right).

influence of the maximum pressure gradient. Here, it should be added that the radial shift observed for this case emphasizes the importance of global simulations.

Figures 9 and 10 depict the mode patterns visualized by the absolute values of the electrostatic perturbation potential in the $s-\theta$-plane and on a flux surface of one field period, respectively. Again, values are normalized to their maximum, and the plots for $M=-1.72 \cdot 10^{-2}(\alpha=+1)$ correspond to the strongly reduced growth rate of the outermost ion-root branch in figure 7 . The right-hand part of figure 9 also gives an impression of the spatial excursions documented in figure 8 since a curved trace of mode positions appears as in an 'afterglow' stretching over the entire poloidal domain.

Figure 10 shows the ITG mode patterns extending along the magnetic field lines and nicely illustrates the absence of a sharply curved helical edge in LHD, thus allowing the ITG modes to move freely into the bottom region of the outer torus.

As discussed with the $\mathrm{W} 7-\mathrm{X}$ case, the reduced growth rates seem to be related to the poloidal (and radial) shifts which occur in the presence of radial electric fields. But the plots also indicate that the ITG modes again get modified, which is finally supported 

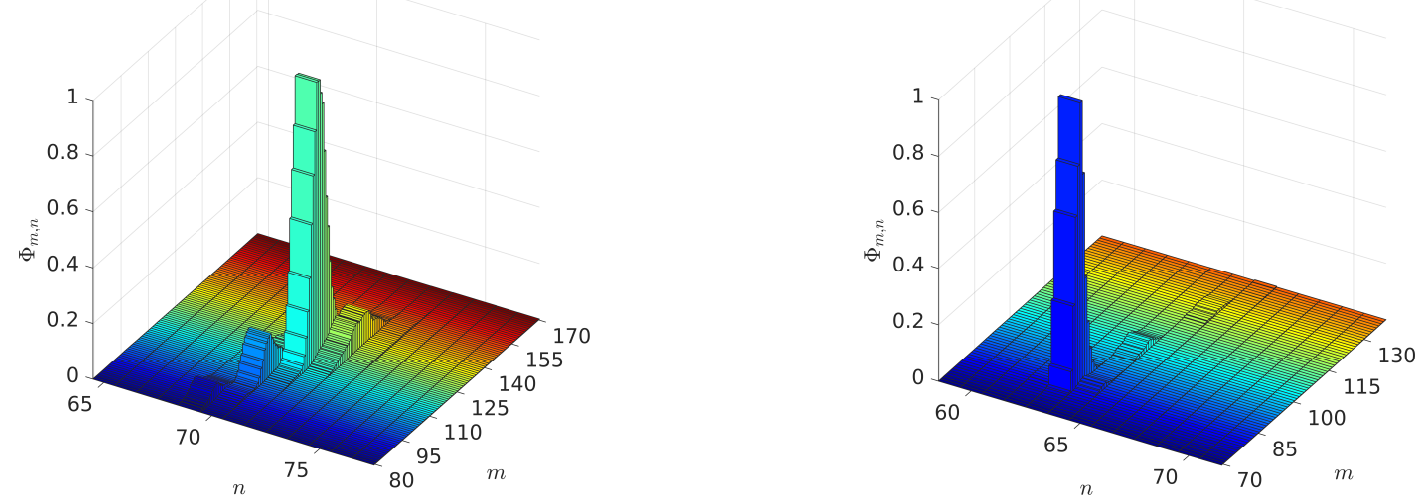

Figure 11. Power spectrum of linear ITG modes in LHD normalized to their maximum as with model A for $M=0$ (left) and $M=-1.72 \cdot 10^{-2}$ (right).

by the result given in figure 11, showing the normalized power spectra (15) obtained with model A for $M=0$ and $M=-1.72 \cdot 10^{-2}$. The spectra are very simple and symmetric - almost similar to tokamaks - with small side bands in $m$ due to a weak coupling of poloidal modes (mode numbers correspond to $k_{\perp} \rho \approx 0.55$ ). Both spectra are dominated by single Fourier components at toroidal mode numbers $n=n_{0}$ with much smaller contributions for neighbouring $n$ indicating a weak coupling of toroidal modes due to the metric properties. For $M=-1.72 \cdot 10^{-2}$, the ITG mode is again shifted towards lower mode numbers.

The clear differences between the ITG mode properties depicted in figures 9 through 11 may also give a hint to the origin of the mode frequencies in figure 7 deviating from the observed Doppler-shift. If we assume the action of a mechanism which 'destroys' the initial mode at some point - e.g. by shearing as described in [4] - so that a formerly subdominant mode with a different frequency now becomes dominant, it seems plausible that this frequency no longer obeys the initially observed Doppler-shift model which can be applied to the case with smaller eld strengths. Referring to the mode frequencies plotted in figure 2, one may also speculate whether the slight deviation from the Doppler-shift rule observed in the ion root of W7-X could also be attributed to such a phenomenon. In this regime, the ITG mode is pushed against the helical edge with its strongly varying metric properties which seem to support the shearing of modes. Thus, the reduction of ITG growth can be seen as the result of a process in which qualitatively different stabilizing mechanisms compete [4].

\section{Summary}

The results presented for W7-X and an LHD-like configuration generally support the common view according to which radial electric fields may lead to a reduction of linear ITG growth rates. Depending on the radial electric field model, an $E \times B$-drift will occur 
which can lead to a spatial displacement of ITG mode structures and a simultaneous modification of characteristic mode properties (growth rate, mode frequency, mode structure, power spectrum). These effects seem to be consistent with the different stabilizing mechanisms described in [4]. Our present results provide an example how the different mechanisms may occur together in fully three-dimensional configurations. W7-X and the LHD-like configuration are seen to lead to qualitatively different ITG modes. Although the different stabilizing mechanisms - the influence of a (mainly) poloidal drift of ITG mode structures as well as the action of mode shearing - can be recognized for both W7-X and LHD, they seem to play a more or less dominant role, depending on the configuration and the strength of the radial electric fields. For W7-X with its pronounced helical edge, the distortion of ITG modes due to the variation of metric properties seems to be dominant. For LHD with its smoothly varying curvature on the outboard side of the torus, the spatial displacement of ITG modes into regions with reduced instability growth can explain the observed reduction of growth rates well. In any case, one should keep in mind that our studies have been performed with simplified pressure profiles which were chosen to lead to radially localized ITG modes. In this sense, the present article deals with localized ITG modes described within a global gyrokinetic model. We regard this approach justified by the circumstance that the effects on such simplified modes can be studied better than those on complex modes, corresponding to realistic pressure profiles. It is possible that the ITG modes resulting from the inclusion of more realistic profiles could be affected even more by the radial electric field. This would be an interesting question to be answered in the future.

\section{Acknowledgements}

This work has been carried out within the framework of the EUROfusion Consortium and has received funding from the European Union's Horizon 2020 research and innovation programme under grant agreement number 633053. The views and opinions expressed herein do not necessarily reflect those of the European Commission.

A part of this work was carried out using the HELIOS supercomputer system at the Computational Simulation Centre of International Fusion Energy Research Centre (IFERC-CSC), Aomori, Japan, under the Broader Approach collaboration between Euratom and Japan, implemented by Fusion for Energy and JAEA.

\section{References}

[1] V. Kornilov, R. Kleiber, R. Hatzky, L. Villard, G. Jost, Gyrokinetic global three-dimensional simulations of linear ion-temperature-gradient modes in Wendelstein 7-X, Phys. of Plasmas 11 (2004) 3196.

[2] Kleiber, R., Hatzky, R., Mishchenko, A., Simulation of Residual Zonal Flow Levels in Stellarators Including a Radial Electric Field, Contributions to Plasma Physics, 50 (2010) 766.

[3] Mishchenko, A., Kleiber, R., Zonal flows in stellarators in an ambient radial electric field, Phys. of Plasmas 19 (2012) 072316. 
[4] L. Villard, A. Bottino, O. Sauter, J. Vaclavik, Radial electric fields and global electrostatic microinstabilities in tokamaks and stellarators, Phys. of Plasmas 9 (2002) 2684.

[5] P. Helander, F. Jenko, R. Kleiber, G. G. Plunk, J. H. E. Proll, J. Riemann, P. Xanthopoulos, Advances in Stellarator Gyrokinetics, Nucl. Fusion 55 (2015) 053030.

[6] J. Riemann, R. Kleiber, M. Borchardt, Global Simulation of Linear Instability in W\%-X and LHD with EUTERPE, 41st EPS Conference on Plasma Physics, Berlin/Germany (2014). http://ocs.ciemat.es/EPS2014PAP/pdf/P1.081.pdf

[7] T. S. Hahm, Nonlinear gyrokinetic equations for tokamak microturbulence, Phys. Fluids 31 (1988) 2670.

[8] R. Hatzky, T. M. Tran, A. Könies, R. Kleiber, S. J. Allfrey, Energy conservation in a nonlinear gyrokinetic particle-in -cell code for ion-temperature-gradient-driven modes in $\theta$-pinch geometry, Phys. of Plasmas 9 (2002) 898.

[9] A. Peeters, D. Strinzi, The effect of a uniform radial electric field on the toroidal ion temperature gradient mode, Phys. of Plasmas 11 (2004) 3748.

[10] H. Ehmler, Y. Turkin, C. D. Beidler, H. Maaßberg, A. Dinklage, T. Klinger and the W7-AS Team, Experimental check of neoclassical predictions for the radial electric field in a stellarator, Nucl. Fusion 43 (2003) L11-L13. 